\title{
Gerontology
}

\section{Robot Therapy: A New Approach for Mental Healthcare of the Elderly - A Mini-Review}

\author{
Takanori Shibata ${ }^{a}$ Kazuyoshi Wada ${ }^{a, b}$ \\ a Intelligent Systems Research Institute, National Institute of Advanced Industrial Science and Technology, Tsukuba, \\ and ${ }^{b}$ Graduate School of System Design, Tokyo Metropolitan University, Tokyo, Japan
}

\section{Key Words}

Mental commitment robot $\cdot$ Robot therapy $\cdot$ Human-robot

interaction · Elderly care $\cdot$ Psychotherapy tic robots, and the seal robot. Finally, we provide examples of robot therapy for elderly people, including dementia patients.

Copyright $\odot 2010$ S. Karger AG, Basel

\begin{abstract}
Mental healthcare of elderly people is a common problem in advanced countries. Recently, high technology has developed robots for use not only in factories but also for our living environment. In particular, human-interactive robots for psychological enrichment, which provide services by interacting with humans while stimulating their minds, are rapidly spreading. Such robots not only simply entertain but also render assistance, guide, provide therapy, educate, enable communication, and so on. Robot therapy, which uses robots as a substitution for animals in animal-assisted therapy and activity, is a new application of robots and is attracting the attention of many researchers and psychologists. The seal robot named Paro was developed especially for robot therapy and was used at hospitals and facilities for elderly people in several countries. Recent research has revealed that robot therapy has the same effects on people as animal therapy. In addition, it is being recognized as a new method of mental healthcare for elderly people. In this mini review, we introduce the merits and demerits of animal therapy. Then we explain the human-interactive robot for psychological enrichment, the required functions for therapeu-
\end{abstract}

\section{Introduction}

Mental healthcare of elderly people is an important issue for caregivers at nursing homes [1]. Elderly people are depressed easily, as they experience difficult situations such as the loss of their family, friends, social roles, and physical functions. Depressive disorders are common among elderly people in nursing homes [2]. In addition, there are people who suffer from other mental diseases like dementia that cause various psychiatric and behavioral disturbances, such as hallucinations, aggression and wandering [3]. Such disorders have a negative impact on the quality of life of both elderly people and their caregivers. Therefore, while trying to communicate with elderly people, caregivers conduct several recreational activities, such as singing songs, coloring, drawing pictures, and origami. However, some people are too embarrassed to sing songs, and others, because of their illness, have difficulty moving their fingers when trying to draw. In addition, caregivers might find communication with the elderly difficult because of lack of common topics.

\section{KARGER}

Fax +4161306 1234

E-Mail karger@karger.ch

www.karger.com (c) 2010 S. Karger AG, Basel

0304-324X/11/0574-0378\$38.00/0

Accessible online at:

www.karger.com/ger
Takanori Shibata, $\mathrm{PhD}$

Intelligent Systems Research Institute

National Institute of Advanced Industrial Science and Technology

1-1-1 Umezono, Tsukuba, Ibaraki 305-8568 (Japan)

Tel. +81 29861 5980, Fax +81 29861 5992, E-Mail shibata-takanori@ aist.go.jp 
Interaction with animals has long been known to be emotionally beneficial to people. In recent years, the effects of animals on humans have been researched and proved scientifically. Friedmann et al. [4] investigated the 1-year survival rate of patients who were discharged from a coronary care unit and found that survival among those who kept pets was higher than among those who did not. Baun et al. [5] reported that the patient's blood pressure dropped when they petted their dog. Garrity et al. [6] studied elderly people who were socially isolated and had lost their partner within the previous year, and they found that the intensity of depression among those who had no pets was higher than among those who did. Lago et al. [7] investigated through telephonic interviews the influences of owning pets on elderly people. They revealed that mortality and attrition were higher for former owners than current owners. Hart et al. [8] studied the social influences of animals on people, and found that the number of friendly approaches by strangers to people with dogs were greater than to people without dogs.

In medical applications, especially in the USA, animalassisted therapy and activities (AAT and AAA) are becoming widely used in hospitals and nursing homes $[9,10]$. AAT has clear goals set out in therapy programs designed by doctors, nurses, or social workers, in cooperation with volunteers. On the other hand, AAA refers to patients interacting with animals without particular therapeutic goals and depends on volunteers. AAT and AAA are expected to have three effects: (1) psychological effect (e.g., relaxation, motivation); (2) physiological effect (e.g., improvement of vital signs), and (3) social effect (e.g., stimulation of communication among inpatients and caregivers).

For example, a hospitalized child, who was in significant pain because of his disease, was afraid to get up and walk around. However, when he was asked to take a therapy dog for a walk, he immediately agreed and walked off happily, as if all his pain had diminished. Moreover, the dog acted as a medium for interaction between him and the other children [11]. In another case, a boy who had been a crack-exposed baby could not speak and walk. However, through interaction with therapy dogs and birds, both his linguistic and motor abilities improved [12].

For AIDS patients it is important to reduce their stress as it is strongly related to the complications of immune deficiency. AAT helps them relax and stay connected with the world [13].

In addition to these effects, AAT and AAA at nursing homes provide rehabilitation to elderly people, and offer laughter and joy to a patient who has a short time to live [14]. AAT reduces loneliness in residents of long-term care facilities [15]. The presence of therapy animals particularly has been useful in reducing agitated behavior, decreasing episodes of verbal aggression and anxiety, and increasing social interaction in institutionalized elderly people suffering from dementia [16-18].

However, most hospitals and nursing homes, especially in Japan, do not allow animals, although they admit the positive effects of AAT and AAA. They are afraid of the negative impact of animals on human beings, such as allergic reactions, infections, bites, and scratches.

This mini review introduces robot therapy and discusses its potential to care for elderly people. The next section describes the new robotics area - human-interactive robots for psychological enrichment. We then explain the required functions for therapeutic robots and the seal robot, Paro. Finally, we provide examples of robot therapy and the conclusions.

\section{Human-Interactive Robots for Psychological Enrichment}

Industrial robots have been used widely in manufacturing industries since the early 1960s. They typically perform welding, assembling, painting, packaging, and palletizing in the automotive manufacturing and other industries. Such robots work very fast and with accuracy, although initially they need to be taught by a human operator and their environment needs to be specially designed for them to accomplish their tasks. Most industrial robots are considered as a potential danger to humans, and therefore are kept isolated from people.

Meanwhile, the rapid development in high technology has produced robots not only for factories but also for our living environment, such as homes, offices, and hospitals. For example, wheelchair robots enable elderly people to easily move outside [19]. Robot suits, which can expand physical capability of humans, are expected to reduce the workload of caregivers [20]. A horseback-riding robot promotes patient's physical strength [21]. Human-interactive robots for psychological enrichment, in particular, are expected to be a new application of robotics and are attracting many researchers and companies [22]. $\mathrm{Hu}-$ man-interactive robots are designed for entertainment, communication (social activity), guidance, education, welfare, mental therapy, and other purposes. Various types of robots, such as humanoid, animal, and robots with unique appearance, have been developed. These robots offer more interaction with humans than industrial robots. They are evaluated not only in terms of objective 
Fig. 1. Objective and subjective measures for evaluating artifacts.

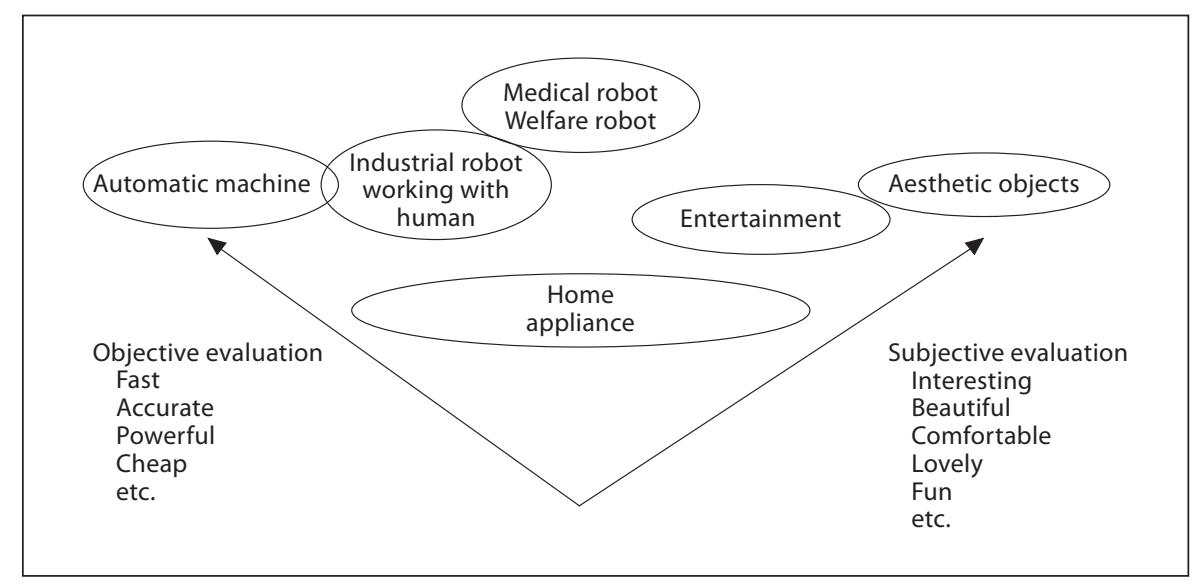

measures, such as speed and accuracy, but also in terms of subjective measures for interacting with humans, such as providing comfort and bringing joy. Robots for entertainment are good examples of the importance of a subjective evaluation of their value (fig. 1).

There are four categories of human-interactive robots for psychological enrichment in terms of their relationship with humans: (1) performance robots; (2) tele-operated performance robots; (3) operation, building, programming, and control robots, and (4) interactive autonomous robots.

\section{Performance Robots}

Performance robots have a long history and they execute movements that express meanings to humans, mostly for fun. Mechanical puppets that could play an organ, draw pictures, and write letters were developed in Switzerland in the 18th century. Karakuri dolls were developed in Japan during the same era to perform dances, magic, and so on. Recently, many performance robots have been used at exhibitions, museums, movies, and amusement parks such as Disneyland and Universal Studios. Recent humanoid robots, such as Honda's ASIMO and Sony's QRIO, can be included in this category [23, 24]. A performance robot can amuse a sizable audience at any time. However, their movements will probably be preprogrammed and mostly repetitive, therefore, they are not usually very interactive with humans. A high degree of complexity in the performance robots is required to keep humans amused.

\section{Tele-Operated Performance Robots}

Tele-operated performance robots are controlled remotely by a hidden operator. Their movements can ap- pear reactive to the humans who interact with them because the operator, based on the audience's actions, sends commands to the robots to simulate reactive behavior. At exhibitions or amusement parks, for example, humantype robots are used as tele-operated performance robots.

\section{Operating, Building, Programming, and}

\section{Controlling Robots}

Humans derive a lot of fun and joy from operating, building, programming, and controlling robots. Moreover, one can watch the performance of the robot that one is operating. A simple example of this is the 'UFO catcher', a stuffed-animal game machine at amusement centers. Building and programming a robot is also included in this category. Contests between robots such as MicroMouse, RoboCup (robot football), and RoboOne (robot wrestling) are popular examples $[25,26]$, as are LEGOMindstorms and I-Blocks. Because building and programming robots can stimulate children's creativity, this activity combines entertainment with education, and is often referred to as 'edutainment' [27, 28].

\section{Interactive Autonomous Robots}

Interactive autonomous robots connect with humans in the physical world. They use verbal and nonverbal communication, depending on the functions of the robots. Contrary to the robots in the other categories, the human-robot interactions are mostly personal. For example, Sony's dog robot, AIBO, which is designed for entertainment, has a mechanical appearance and attracts people's interest using nonverbal communication [29]. The communication robot, ifbot, produces conversation using facial expressions and a large number of prepared conversation scenes [30]. The human-friendly informa- 


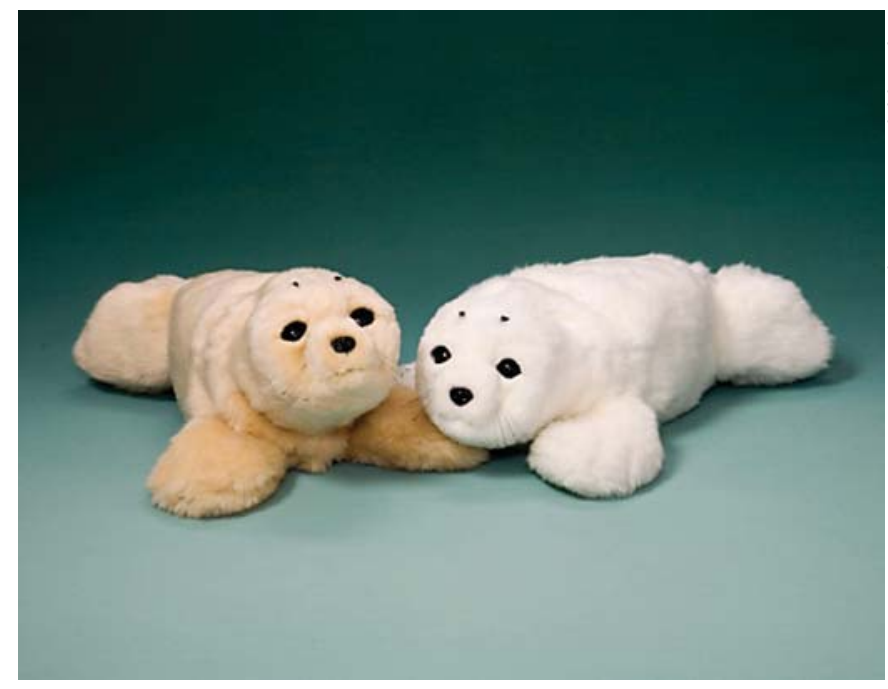

Fig. 2. Seal robot, Paro.

tion terminal, PaPeRo, can control home electric appliances, collect information via the Internet by voice command, and even entertain people by dancing and playing games [31]. Guide robots in museums and exhibitions [32] and mental commitment robots described in this paper also belong to this category.

In the area of welfare and mental therapy, Shibata et al. [22, 33-49] studied and developed a mental commitment robot, which aims to engender mental effects, such as pleasure and relaxation, in its role as a personal robot. They also propose a robot therapy, which uses robots as substitutes for animals in AAT and AAA. Robot therapy targets people in medical and welfare institutions where animals are not allowed. A seal-type mental commitment robot, named Paro (fig. 2), was developed especially for robot therapy and is used at pediatric hospitals and facilities for elderly people in several countries. Recent research has revealed that robot therapy has the same effects on people as AAT. Robot therapy is recognized, in particular, as a new method of mental healthcare for elderly people (including dementia patients).

\section{Therapeutic Robot}

\section{Required Functions}

In robot therapy, it is important to stimulate people's knowledge and experiences of animals through interaction with the robots and to bring out their feelings when they are interacting with animals. Therefore, shapes, feelings of touch, autonomous behavior, and responses that mimic animals are the features that are required to be present in the robots. In addition, the devices are used not only at people's homes but also at hospitals and nursing homes. Many people who have lost their physical strength and healing capability due to aging and illness are expected to interact with the robots. Therefore, the robots should be easily accepted by people and also be harmless and hygienic. A concern with such robots is that individuals are expected to physically interact by touching and hugging them, and therefore there exists a possibility of people being harmed. Furthermore, in case of the robot being used by people with deteriorated immune systems such as leukemia patients in hospitals, the robot may possibly transmit germs. Furthermore, some people visit medical welfare facilities for a few hours at a time for day care and ambulant treatment, but some stay or are hospitalized for years (e.g., nursing homes and long-term care). Therefore, the robots for long-term therapies have to sustain interaction with people in their daily lives.

These robots would be used by doctors, nurses, therapists, caregivers, and volunteers during a certain period of time. In addition, the users would play with them whenever they want. Therefore, it is important that these robots are designed in such a manner that anyone can operate them, and that no specialized knowledge is required to do so.

\section{Mental Commitment Robot, Paro}

Mental commitment robots are not intended to offer people physical work or service [22, 33-49]. Their function is to engender mental effects, such as pleasure and relaxation, in their role as personal robots. These robots act independently with a purpose and with motives while receiving stimulation from the environment, like living organisms. Actions that manifest themselves during interactions with people can be interpreted as if the robots had hearts and feelings.

Mental commitment robots can stimulate the different senses of human beings through physical interaction. Therefore, the primary characteristic of mental commitment robots is nonverbal communication. A basic psychological experiment was conducted on the subjective interpretation and evaluation of robot behavior following human-robot interactions. This study showed the importance of appropriately stimulating the human senses and extracting associations. Sensor systems, such as visual, aural, and tactile senses for robots, were studied and developed. A plane soft tactile sensor was developed to cov- 
er the robot to enhance bodily contact between people and the robot [42]. This sensor can detect position and force when people touch the robot, and at the same time, it is soft to touch.

The shapes of the animal robots are classified into three categories: (1) familiar animals (e.g., dog, cat); (2) nonfamiliar animals (e.g., seal), and (3) imaginary animals or characters.

Dog, cat, and seal robots were developed as models. The robot operates using the three elements of its internal states, sensory information from its sensors, and its own diurnal rhythm (morning, daytime, and night) to perform various activities during its interaction with people.

Subjective evaluations of cat and seal robots were conducted using a questionnaire $[35,36]$. People valuated both robots highly. However, the subjects complained about the softness and the reactions of the cat robot in comparison with their knowledge of real cats. On the other hand, most people do not have much knowledge about seals, and hence were unable to compare the seal robot with their knowledge of seals. Therefore, the seal robot's evaluation was higher after the interaction. These results revealed that more people accepted the unfamiliar animal shape. Cross-cultural studies on the subjective evaluation of the seal robot were conducted in seven different countries: Japan, UK, Sweden, Italy, Korea, Brunei, and the USA [40,49]. The data were obtained from over 1,800 respondents. The subjective evaluation provided overall high scores, and further revealed that the seal robot could be widely accepted despite cultural and religious differences.

The seal-type mental commitment robot, Paro, was designed for therapy. For this purpose, it was functionally designed to be soft and evoke a feeling of warmth. Each seal robot was trimmed in artificial fur and eyelashes were sewn on it by craft workers to achieve a high quality. In addition, its artificial fur was antibacterial and dirt-resistant, and would not fall out. An electromagnetic shield was provided to the internal circuit to prevent any ill effect on heart pacemakers. The withstand voltage test, drop test, 100,000 times stroking test, and a longterm clinical test over the years confirmed that Paro is highly safe and durable. It was designed to be simple enough for anyone to be able to operate. Paro has only one on/off switch for power, and a pacifier-type charger. Learning the functions of its name and behavior allows its users to gradually build a relationship with it, thus preventing them from losing interest and in turn encouraging them to show their affection for Paro. In addition, the baby harp seal was ecologically investigated to model its
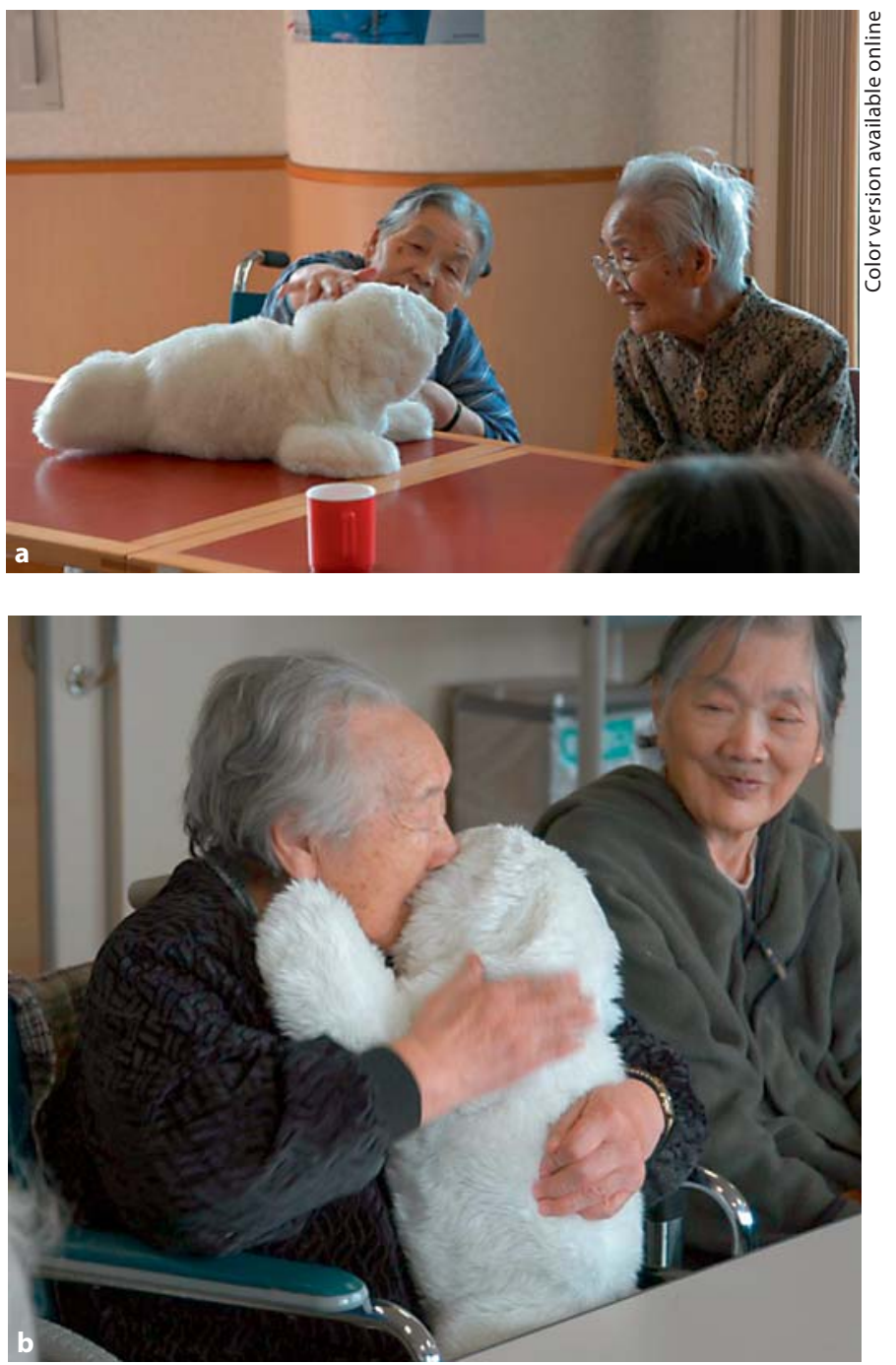

Fig. 3. Elderly people (a) speaking and stroking and (b) hugging and kissing the seal robot, Paro.

liveliness and cuteness in the robot; actual baby seal calls were sampled and used.

\section{Robot Therapy}

Robot therapy using the seal robot is conducted at hospitals and nursing homes in many countries: Japan, Sweden, Denmark, Italy, the USA, etc. Robot therapy consists of robot-assisted therapy programs designed by doctors, nurses, and social workers, and also robot-assisted activity, which allows patients to interact with robots without any particular therapeutic goals. Such activities do not depend on volunteers, but are conducted by facility staff. Robot-assisted therapy is mainly conducted at medical 
facilities, such as hospitals and clinics, while robot-assisted activity is conducted at welfare facilities, such as nursing homes (fig. 3).

\section{Robot Therapy for Elderly People}

As an example for robot therapy, Paro was used to assist elderly people at a day service center $[38,39,43]$. To investigate the effects of seal robots on elderly people, their mood was evaluated using face scales [50] and questionnaires. Changes in their reactions to stress were measured by the hormones in urine: 17-ketosteroid sulfate (17-KS-S) and 17-hydroxycorticosteroids (17-OHCS) [51, 52]. In addition, the stress that the nursing staff experienced was investigated by questionnaires, i.e. the burnout scale [53]. The day service center was provided with seal robots for 5 weeks, as a result, the feelings of the elderly people improved by their interaction with the robots. Urine samples showed that their ability to overcome stress also improved. Moreover, the stress levels of the nursing staff decreased because the elderly people required less supervision while interacting with the robots.

A long-term experiment was conducted at a health service facility starting in August 2003 [45]. Approximately 10 people joined the interaction with Paro for $1 \mathrm{~h}$, twice a week. One or two caregivers managed the interaction with Paro. To investigate the effects of Paro, face scale and geriatric depression scales [54] were used. The results showed that the feelings of the elderly people improved over the year, and depression in the participants was also reported to have been reduced. Caregivers commented that interaction with Paro made the elderly people laugh and become more active. Their facial expressions changed, softened, and brightened. On the day of the activity, they looked forward to interacting with Paro, sitting down in their seats even before the interaction session began. Some people who usually stayed in their rooms came out and willingly joined the activity. In addition, Paro encouraged people to communicate with each other as well as with the caregivers by becoming their common topic of conversation. Thus, the general atmosphere became brighter. Even now, these elderly people enjoy playing with Paro.

In another example, Paro was introduced in the public area of a care house, a type of communal housing for elderly people, and was activated for over $9 \mathrm{~h}$ each day for the researchers to investigate the effects of free interaction with it [47]. To examine the psychological and social effects, each subject was interviewed and his or her social network analyzed. In addition, the activities of the residents in public areas were video recorded. For physiolog-

Robot Therapy - A New Approach for Mental Healthcare of the Elderly ical analysis, residents' hormones in urine, 17-KS-S and 17-OHCS, were analyzed. The results indicate that interaction with Paro increased their social interaction. Furthermore, the urine tests showed that the reactions of the subjects' vital organs to stress improved after interacting with Paro.

\section{Effects on Patients with Dementia}

Dementia is an important problem in elder care. According to Alzheimer's Disease International (ADI), an estimated 24.4 million people suffer from dementia worldwide, and the number will increase to 82 million by 2040. Dementia is a progressive, disabling neurological condition that occurs in a wide variety of diseases. The most common cause of dementia is Alzheimer's disease, which accounts for approximately half of the people with dementia. Other causes include vascular disease, Lewy body dementia, and many other diseases [55]. Psychiatric and behavioral disturbances, such as personality changes, hallucinations, paranoid ideas, aggression, wandering, and incontinence are common features of dementia and are the leading causes of the need for long-term care [3]. Donepezil, physical exercise, and diet cure are expected to slow the progress of dementia [56], but unfortunately, there is no permanent cure for dementia at present. Recent data suggest that art, music, and learning, which stimulate patients' emotions and brain, can slow its progression once it has begun [57-59]. However, there is room for improvement in all such treatments.

As for the interaction between Paro and dementia patients in nursing homes, behavioral improvements were observed in several cases. For example, a patient who moaned continuously was able to relax and then started to talk to the therapist [46]. After playing with Paro, another patient who often tried to return home stopped doing so; her wandering symptom was improved.

Robot therapy for dementia patients was conducted at a neurosurgery clinic to investigate the physiological influences of the treatment [48]. Diagnosis Method of Neuronal Dysfunction (DIMENSION) was used to record each patient's EEG before and after 20 min of robot therapy [60]. In addition, a questionnaire concerning each subject's impression of Paro was conducted. The results showed that $50 \%$ of 14 valid subjects' condition of cortical neurons activity improved by interacting with Paro. This is especially true for patients who liked Paro very much.

In Japan, the cost of care for a patient with dementia is about USD 40,000 per year, and the patient's life expectancy is about 8 years. This represents an enormous burden on the municipalities that provide long-term care in- 
surance. Useful and convenient methods for the prevention of dementia are urgently needed. Paro has been commercialized since 2005. The price is about EUR 4,500 . However, the running costs are almost only a recharging fee of the battery, as it is designed to have enough durability over 10 years of use. Paro can be used even without a specially trained therapist. Several municipalities in Japan anticipate the effects of Paro and support its introduction. For example, Nanto City, Toyama, bought eight Paros and introduced them to day service centers in the city. Tsukuba City, Ibaraki, established a subsidy for purchasing Paro.

Meanwhile, a dementia care center in Copenhagen, Denmark, investigated the effects of robot therapy on dementia patients as part of the national project 'Be-Safe'. Twelve Paros were introduced to ten different places. The results obtained from the 7-month clinical trial showed that Paro had positive effects on the patients. Based on the results, the Danish government decided to introduce 1,000 units of Paros to nearly all of the elderly care facilities in Denmark.

\section{Other Research}

Robot therapy, using commercially available animaltype robots, such as AIBO and NeCoRo [61], has been attempted [62-64]. For example, Libin and Libin [62] introduced NeCoRo to a nursing home and observed the patients' interaction. Kanamori et al. [63] examined the effects of AIBO on elderly people in a nursing home. By measuring the hormones in saliva, they found that stress decreased after a 1-hour interaction with AIBO, and that loneliness was reduced after 20 sessions over a 7-week period. Tamura et al. [64] compared the exposure of patients to AIBO with the effect of exposure to a toy dog. They found that AIBO did not encourage interaction much, and required more intervention from an occupational therapist.

Because they are not designed for therapy, these commercially produced robots easily break while interacting with people. Therefore, it is difficult to use them in longterm situations.

\section{Conclusion}

Various robots have been developed and are being introduced in our lives as commercial products. Each robot is designed for a specific purpose. The seal-type mental commitment robot, Paro, whose goal is to enrich daily life and heal human minds, is designed to maintain long-

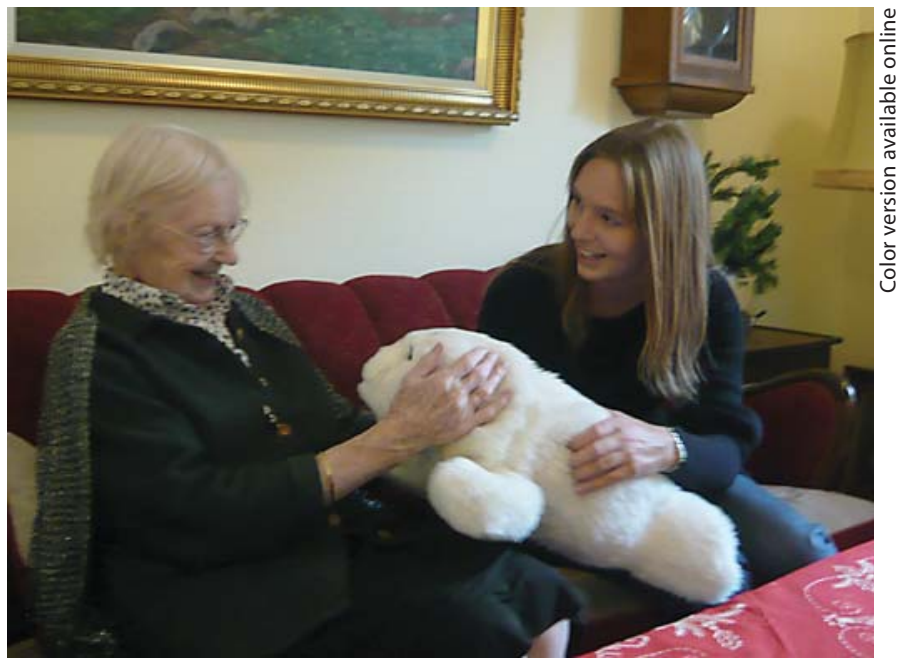

Fig. 4. Social mediator, Paro, between elderly person and caregiver.

term interaction with people and provide them with psychological, physiological, and social benefits.

Robot therapy, mental healthcare using animal-type robots, is an emerging field. The results of exploratory experiments showed that Paro has a great potential to provide mental healthcare for elderly people. However, more subjects and a control group are necessary to scientifically verify its effects. Further experiments will be conducted in this regard.

As for ethical issues, the experiments using Paro that were mentioned above were conducted under the ethical committee of each organization. Only the people who and whose relatives agreed to robot therapy joined. In addition, people might worry that elderly people will spend time alone with Paro when the caregiver leaves them to interact with it. But, the opposite is true. Paro would be a social mediator among them as a common topic for them, and encourage them to communicate with each other (fig. 4). Currently, the method of robot therapy is left to the caregivers and its effects are influenced by them. To ensure Paro's potential, developing the effective ways to use it is the next step.

Currently, approximately 1,500 Paros have been sold in the world (about 1,300 in Japan, 100 in Denmark, and 100 in other countries). Paro is highly accepted and similar psychological effects are shown in each country. However, when beginning to use it, there are some cultural differences in the acceptance of Paro. People in Europe tended to note the effects of interaction with Paro 
and accepted it as a therapeutic tool, because AAT has been used widely in hospitals and nursing homes. Meanwhile, in Asian countries, AAT is not commonly accepted although many people have had pets recently. They tended to accept Paro as their companion more than as a therapeutic tool. In the USA, they tended to accept Paro as a therapeutic tool as well as a companion [49]. Therefore, it is important to introduce Paro in a suitable manner based on the cultural differences.

Robot therapy in medical and welfare facilities is spreading in our society. Further studies will be conducted to integrate robot therapy into our societies at large.

\section{References}

1 Daies B, Knapp M (eds): Old People's Homes and the Production of Welfare. Boston, Routledge \& Kegan Paul, 1981.

2 WPA/PTD Educational Program on Depressive Disorders, Module 3: Depressive Disorders in Older Persons (http://www.wpanet. org/education/ed-program-guidelines.sht$\mathrm{ml}$ ).

3 Alzheimer's Disease International: Psychiatric and Behavioural Disturbances in Dementia. ADI Factsheet 1999, No 7

-4 Friedmann E, Katcher A, Lynch J, Thomas S: Animal companions and one-year survival of patients after discharge from a coronary care unit. Public Health Rep 1980;95:307312.

5 Baun M, Bergstrom N, Langston N, Thoma L: Physiological effects of human/companion animal bonding. Nurs Res 1984;33:126129.

6 Garrity T, Stallones L, Marx M, Johnson T: Pet ownership and attachment as supportive factors in the health of the elderly. Anthrozoos 1989;3:35-44.

7 Lago D, Delaney M, Miller M, Grill C: Companion animals, attitudes toward pets, and health outcomes among the elderly: a longterm follow-up. Anthrozoos 1989;3:25-34.

8 Hart L, Hart B, Bergin B: Socializing effects of service dogs for people with disabilities. Anthrozoos 1987;1:41-44.

9 Standards of Practice for Animal-Assisted Activities and Therapy. Renton/WA, Delta Society, 1996.

10 Fine AH: Handbook on Animal-Assisted Therapy: Theoretical Foundations and Guidelines for Practice, ed 2. New York, Academic Press, 2006.

11 Kale M: Kids and animals. Inter Actions 1992;10:17-21.

12 Animal-Assisted Therapy and Crack Babies: A New Frontier. Delta Society Newsletter 1991, No 1.

13 Haladay J: Animal-assisted therapy for PWAs - bringing a sense of connection. AIDS Patient Care 1989;38-39.

14 Gammonley J, Yates J: Pet projects animal assisted therapy in nursing homes. J Gerontol Nurs 1991;17:12-15.
5 Banks MR, Banks WA: The effects of animal-assisted therapy on loneliness in an elderly population in long-term care facilities. J Gerontol A Biol Sci Med Sci 2002;57:428432.

16 Richeson NE: Effects of animal-assisted therapy on agitated behaviors and social interactions of older adults with dementia. Am J Alzheim Dis Other Dement 2003;18: 353358.

17 Fick KM: The influence of an animal on social interactions of nursing home residents in a group setting. Am J Occup Ther 1993;47: 529-534.

18 Fritz CL, Farver TB, Kass PH, Hart LA: Association with companion animals and the expression of noncognitive symptoms in Alzheimer's patients. J Nerv Ment Dis 1995; 183:459-463.

19 Matsumoto O, Komoriya K, Hatase T, Nishimura H: Autonomous Traveling Control of the 'TAO Aicle' Intelligent Wheelchair. Proc IEEE/RSJ Int Conf IROS, 2006, pp 4322-4327.

20 Website of robotic suit, HAL. http://www. cyberdyne.jp

21 Website of horseback riding robot, JOBA. http://panasonicjp/fitness

22 Shibata T: An overview of human interactive robots for psychological enrichment. Proc IEEE 2004;92:1749-1758.

23 Hirai K: Humamoid robot and its applications. Proc IARP Int Conf on Humanoid and Human Friend Robot 1998;1:1-4.

24 Kuroki Y, Ishida T, Yamaguchi J, Fujita M, Doi T: A small biped entertainment robot. J Robot Mechatron 2002;14:7-11.

25 Kitano H, et al: The Robocup '97 Synthetic Agents Challenge; in RoboCup-97: Robot Soccer World Cup I. New York, Springer, 1998, pp 62-73.

26 Robo-One Official Homepage. http://www. robo-one.com

27 Druin A, Hendler J (eds): Robots for Kids; Exploring New Technologies for Learning. San Francisco, Morgan Kaufmann, 2000.

28 Lund H: Modern artificial intelligence for human-robot interaction. Proc IEEE 2004; 92:1821-1838.

29 Fujita M: On activating human communications with pet-type robot AIBO. Proc IEEE 2004;92:1804-1813.
30 Website of ifbot. http://www.business-design.co.jp

31 Website of PaPeRo. http://www.incxneccojp/robot/robotcenter.html

32 Bischoff R, Graefe V: Hermes - a versatile personal robotic assistant. Proc IEEE 2004; 92:1759-1779.

33 Shibata T, Inoue K, Irie R: Emotional robot for intelligent system - artificial emotional creature project. Proc IEEE RO-MAN, 1996, pp 466-471.

34 Shibata T, Irie R: Artificial emotional creature for human-robot interaction - a new direction for intelligent system. Proc IEEE/ ASME Int Conf AIM, 1997, No 47.

35 Shibata T, Tashima T, Tanie K: Subjective Interpretation of emotional behavior through physical interaction between human and robot. Proc IEEE Int Conf SMC 1999, pp 10241029.

36 Shibata T, Tanie K: Influence of a-priori knowledge in subjective interpretation and evaluation by short-term interaction with mental commit robot. Proc IEEE/RSJ Int Conf IROS 2000;1:169-174.

37 Shibata T, Mitsui T, Wada K, Touda A, Kumasaka T, Tagami K, et al: Mental commit robot and its application to therapy of children. Proc IEEE/ASME Int Conf AIM, 2001, pp 1053-1058.

38 Shibata T, Wada K, Saito T, Tanie K: Robotassisted activity for senior people at day service center. Proc Int Conf ITM, 2001, pp 7176.

39 Saito T, Shibata T, Wada K, Tanie K: Examination of change of stress reaction by urinary tests of elderly before and after introduction of mental commit robot to an elderly institution. Proc Int Symp AROB 2002;1:316-319.

40 Shibata T, Mitsui T, Wada K, Tanie K: Subjective evaluation of seal robot, Paro - tabulation and analysis of questionnaire results. J Robot Mechatron 2002;14:13-19.

41 Wada K, Shibata T, Saito T, Tanie K: Psychological and social effects in long-term experiment of robot-assisted activity to elderly people at a health service facility for the aged. Proc IEEE/RSJ Int Conf IROS, 2004, pp 3068-3073. 
42 Shibata T: Ubiquitous surface tactile sensor. TExCRA 2004, p 56.

43 Wada K, Shibata T, Saito T, Tanie K: Effects of robot-assisted activity for elderly people and nurses at a day service center. Proc IEEE 2004;92:1780-1788.

44 Marti P, Palma V, Pollini A, Rullo A, Shibata T: My gym robot. Proc Symp Robot Companions, 2005, pp 64-73.

45 Wada K, Shibata T, Saito T, Sakamoto K, Tanie K: Psychological and social effects of one year robot assisted activity on elderly people at a health service facility for the aged. Proc IEEE ICRA, 2005, pp 2796-2801.

46 Marti P, Bacigalupo M, Giusti L, Mennecozzi C, Shibata T: Socially assistive robotics in the treatment of behavioural and psychological symptoms of dementia. Proc Int Conf BioRob, 2006, pp 483-488.

$\checkmark 47$ Wada K, Shibata T: Living with seal robots - its socio-psychological and physiological influences on the elderly in a care house. IEEE Trans Robot 2007;23:972-980.

48 Wada K, Shibata T, Musha T, Kimura S: Robot therapy for elders affected by dementia. IEEE Eng Med Biol Mag 2008;27:53-60.
49 Shibata T, Wada K, Ikeda Y, Sabanovic S: Cross-cultural studies on subjective evaluation of seal robot. Adv Robot 2009;27:443458.

50 Lorish CD, Maisiak R: The face scale: a brief, nonverbal method for assessing patient mood. Arthritis Rheum 1986;29:906-909.

51 Selye H: Stress and aging. J Am Geriatr Soc 1970;18:669-676.

52 Nishikaze O, et al: Distortion of adaptation (wear \& tear and repair \& recovery) - urine 17-KS sulfates and psychosocial atressin. Humans-Job Stress Res 1995;3:55-64.

53 Pines A: The Burnout Measure. National Conference on Burnout in the Human Services, Philadelphia, November 1981.

54 Yesavage JA: Geriatric depression scale. J Psychopharmacol Bull 1988;24:709-711.

55 Alzheimer's Disease International: The Prevalence of Dementia. ADI Factsheet 1999, No 3

56 Andrade C, Radhakrishnan R: The prevention and treatment of cognitive decline and dementia: an overview of recent research on experimental treatments. Indian J Psychiatry 2009;51:12-25.

57 Kimura S, Kaneko K, Nishida K, Sekine K, Musha T, Oh M: Effectiveness of the creative art therapy by using the Diagnosis Method of Neuronal Dysfunction (DIMENTION) Report. Kansei Fukushi Res Cent 2002;3: 195-200.
58 Brotons M, Koger S: The impact of music therapy on language functioning in dementia. J Music Ther 2000;37:183-195.

59 Kawashima R: Cognitive rehabilitation for Alzheimer disease - the learning therapy. Rinsho Shinkeigaku 2002;45:864-866.

60 Musha T, Asada T, Yamashita F, Kinoshita T, Chen Z, Matsuda H, et al: A new EEG method for estimating cortical neuronal impairment that is sensitive to early stage Alzheimer's disease. Neurophysiol Clin 2002;113: 1052-1058.

61 Website of NeCoRo. http://www.necorocom/home.html

62 Libin A, Libin E: Person-robot interactions from the robopsychologists' point of view: the robotic psychology and robotherapy approach. Proc IEEE 2004;92:1789-1803.

63 Kanamori M, Suzuki M, Tanaka M: Maintenance and improvement of quality of life among elderly patients using a pet-type robot. Nippon Ronen Igakkai Zasshi 2002;39: 214-218.

64 Tamura T, Yonemitsu S, Itoh A, Oikawa D, Kawakami A, Higashi Y, et al: Is an entertainment robot useful in the care of elderly people with severe dementia? J Gerontol A Biol Sci Med Sci 2004;59A:83-85. 